\title{
ORIGINAL RESEARCH \\ The Effect of Intracranial Stent Implantation on the Curvature of the Cerebrovasculature
}

\author{
R.M. King \\ J.-Y. Chueh \\ I.M.J. van der Bom \\ C.F. Silva \\ S.L. Carniato \\ G. Spilberg \\ A.K. Wakhloo \\ M.J. Gounis
}

\begin{abstract}
BACKGROUND AND PURPOSE: Recently, the use of stents to assist in the coiling and repair of wide-neck aneurysms has been shown to be highly effective; however, the effect of these stents on the $\mathrm{RC}$ of the parent vessel has not been quantified. The purpose of this study was to quantify the effect of intracranial stenting on the RC of the implanted artery using 3D datasets.
\end{abstract}

\begin{abstract}
MATERIALS AND METHODS: Twenty-four patients receiving FDA-approved neurovascular stents to support coil embolization of brain aneurysms were chosen for this study. The stents were located in the ICA, ACA, or MCA. We analyzed C-arm rotational angiography and contrast-enhanced cone beam CT datasets before and after stent implantation, respectively, to ascertain changes in vessel curvature. The images were reconstructed, and the vessel centerline was extracted. From the centerline, the RC was calculated.
\end{abstract}

RESULTS: The average implanted stent length was $25.4 \pm 5.8 \mathrm{~mm}$, with a pre-implantation $\mathrm{RC}$ of $7.1 \pm$ $2.1 \mathrm{~mm}$ and a postimplantation $\mathrm{RC}$ of $10.7 \pm 3.5 \mathrm{~mm}$. This resulted in a $3.6 \pm 2.7 \mathrm{~mm}$ change in the RC due to implantation $(P<.0001)$, more than a $50 \%$ increase from the pre-implantation value. There was no difference in the change of RC for the different locations studied. The change in $\mathrm{RC}$ was not impacted by the extent of coil packing within the aneurysm.

CONCLUSIONS: The implantation of neurovascular stents can be shown to have a large impact on the $\mathrm{RC}$ of the vessel. This will lead to a change in the local hemodynamics and flow pattern within the aneurysm.

ABBREVIATIONS: $\mathrm{ACA}=$ anterior cerebral artery; $\mathrm{CBCT}=$ cone-beam $\mathrm{CT}$; $\mathrm{CFD}=$ computational fluid dynamics; $3 D R A=3 D$ rotational angiography; $\mathrm{NiTi}=$ nitinol; $R C=$ radius of curvature
S tents have emerged as a new tool to treat intracranial aneurysms. First approved by the FDA in 2002, the Neuroform stent (formerly Boston Scientific, Natick, Massachusetts) was the first self-expanding stent indicated for intracranial use. Before its approval, other cardiac stents were sporadically used when no other treatment option was available. $^{1,2}$ The major limitation of these stents is that they are often too rigid to navigate beyond the tortuous ICA siphon. A number of studies have indicated that the new generation of self-expanding neurovascular stents provides valuable adjunctive tools in the treatment of wide-neck aneurysms. ${ }^{3-5}$ However, the exact mechanism by which stents aid in aneurysmal healing is unknown.

Currently, there are 3 mechanisms by which stents are thought to promote this healing. First, the stent may limit the flow into the aneurysm by allowing tight packing of the aneurysm neck, ${ }^{6}$ leading to stagnation and stable clot formation within the aneurysmal sac. Second, the endothelialization of the stent struts may induce neointimal tissue growth over the aneurysm neck,

Received October 6, 2011; accepted after revision January 5, 2012.

From the Department of Radiology (R.M.K., J.-Y.C., I.M.J.v.d.B., C.F.S., G.S., A.K.W., M.J.G.), New England Center for Stroke Research, University of Massachusetts Medical School, Worcester, Massachusetts; and Research and Development (S.L.C.), Stryker Neurovascular, Fremont, California.

This work was supported by Stryker Neurovascular.

Please address correspondence to Matthew J Gounis, PhD, Department of Radiology, University of Massachusetts, New England Center for Stroke Research, 55 Lake Ave North, SA-107R, Worcester, MA 01655; e-mail: matt.gounis@umassmed.edu

Indicates open access to non-subscribers at www.ajnr.org

http://dx.doi.org/10.3174/ajnr.A3062 leading to its exclusion from the circulation, as has been shown in a single human case following autopsy ${ }^{7}$ and in an animal model. ${ }^{8}$

Finally, the change in the geometry of the blood vessel from stent implantation may have an effect on the local hemodynamics. Studies on hemodynamic and blood vessel characteristics have shown that aneurysms are more likely to develop in areas of higher wall shear stress, such as the outer wall of curved vessels or bifurcations. ${ }^{9}$ This was further demonstrated in clinical reviews. ${ }^{10}$ It has also been shown that ICA aneurysms form at the curvature points and that rupture takes place more often in bends with a smaller RC. ${ }^{11}$ Although the curvature and diameter of the ICA siphon are available, ${ }^{12}$ changes in curvature of intracranial arteries due to implantation of self-expanding NiTi stents are little known.

It has been documented that stent deployment within the coronary arteries results in a significant change in vessel curvature of approximately $20 \% .^{13,14}$ Due to anatomic differences between coronary and neurovasculature as well as different stent properties, these results cannot be translated to cerebrovascular self-expanding stents. Most recently, Huang et $\mathrm{al}^{15}$ observed an increase in the angle between the Al segment and the ipsilateral or contralateral A2 segment after stent-assisted coil embolization of anterior communicating artery aneurysms. The measurements were acquired from selected projection angiograms. The major limitations of this method, as mentioned by the authors, included the difficulties in describing $3 \mathrm{D}$ vascular geometry changes by using $2 \mathrm{D}$ angiographic datasets and establishing uniform measurement standards. In this study, we aimed to quantify the change in the $\mathrm{RC}$ of the parent artery induced by the implantation of an intracranial stent in $3 \mathrm{D}$. 

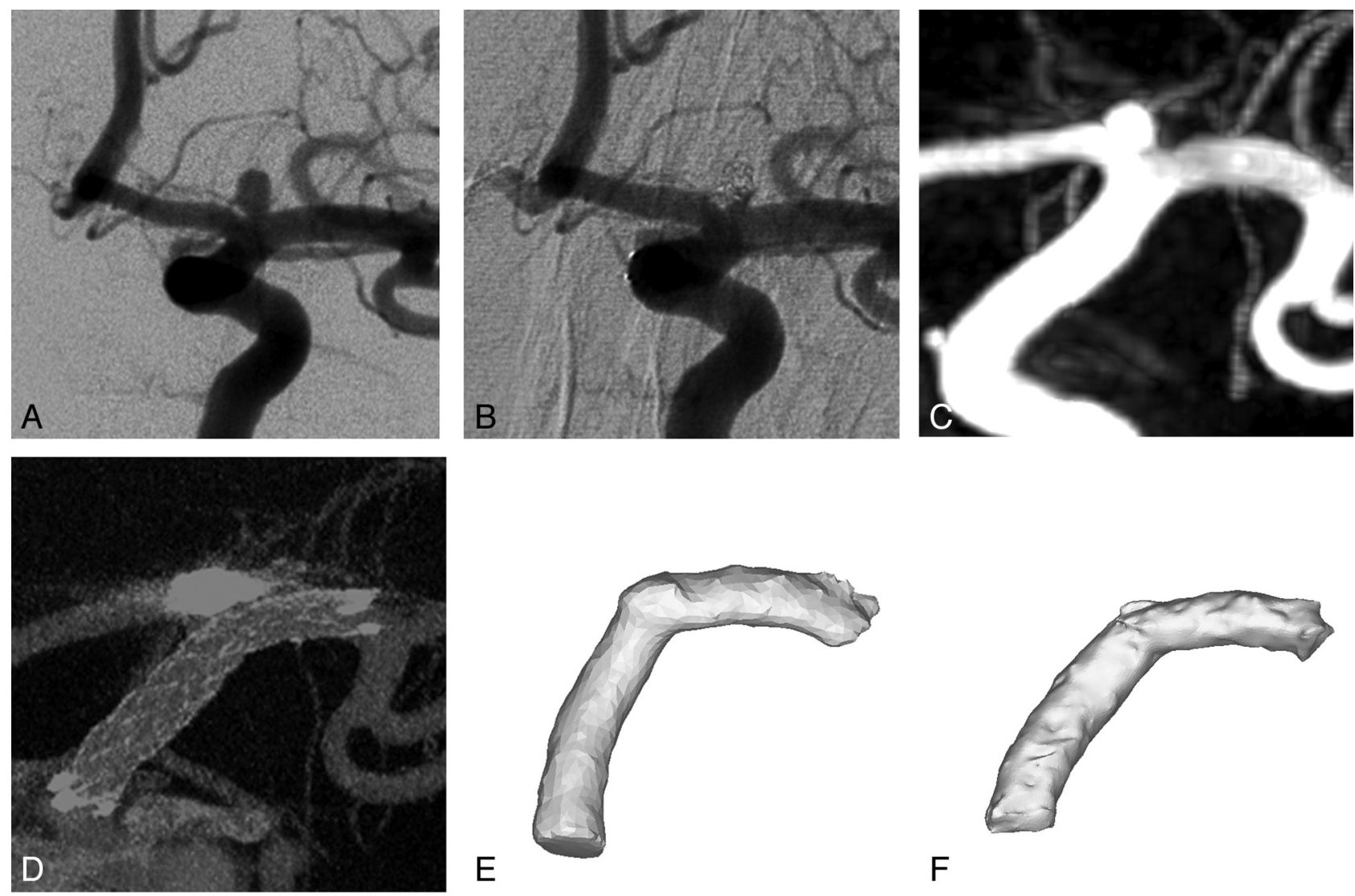

$\mathrm{F}$

G
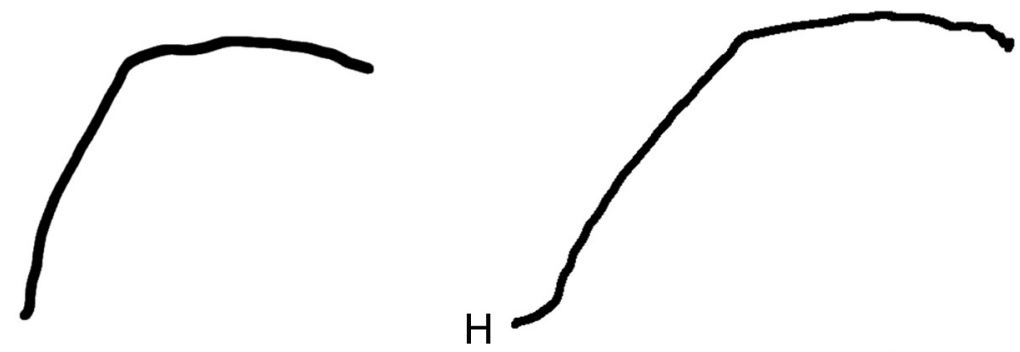

Fig 1. Illustrative case of a 61-year-old female with family history of aneurysmal subarachnoid hemorrhage. MR angiography revealed a left ICA terminus aneurysm. Catheter angiography demonstrated a $2.5 \mathrm{~mm}$ aneurysm having a $2 \mathrm{~mm}$ neck $(A$, frontal oblique projection) that subsequently underwent stent assisted coil embolization ( $B$, frontal oblique projection). Pre-embolization 3DRA $(C)$ and post-embolization CBCT $(D)$ are used to isolate a 3D model of the stented artery (pre-embolization, E; post-embolization, $F$ ). The centerlines of the stented vascular segment pre and post stent-assisted coiling are extracted in $G$ and $H$, respectively.

\section{Materials and Methods}

\section{Imaging}

Patients were retrospectively selected on the basis of the following criteria: 1) the availability of pretreatment 3DRA and postimplantation contrast-enhanced CBCT (Allura FD20/20; Philips Healthcare, Best, the Netherlands) of the stented vessel segment, 2) the use of a self-expanding NiTi intracranial stent (Enterprise VRD; Codman Neurovascular, Raynham, Massachusetts; or Neuroform; Stryker Neurovascular, Fremont, California), 3) the location of the stent within the anterior intracranial circulation, and 4) streak artifacts caused by the platinum coils within the associated aneurysm that did not interfere with post-imaging processing to segment the stented vessel. We reviewed our data base of 238 aneurysms that received stent-assisted coil embolization and found 24 cases that met these criteria. Specifically, postembolization high-resolution CBCT was not available in 137 patients, pretreatment 3DRA was not available in 7 patients, 41 cases were excluded due to location of the stent within the posterior circulation, and 39 cases were excluded due to streak artifacts obscuring the implanted vessel segment. Note that some patients met $>1$ exclusion criterion.

Our current imaging protocol is to acquire 3DRA before treatment and contrast-enhanced cone-beam CT immediately after stent deployment to assess the relationship of the vessel and device. 3DRA imaging was performed by acquiring 122 projection images over a $210^{\circ}$ arc (rotation time, 4.7 seconds) at approximately $88 \mathrm{kVp}$ and a total of $200 \mathrm{mAs}$ (Fig 1C). During 3DRA acquisitions, contrast (iopamidol 51\%, Isovue; Bracco Diagnostics, Princeton, New Jersey) was injected through the $6 \mathrm{~F}$ guide catheter positioned in the cervical ICA 
via a coupled power injector (Mark V ProVis; Medrad, Indianola, Pennsylvania) at a rate of $5 \mathrm{~mL} / \mathrm{s}$ for a total of $30 \mathrm{~mL}$ with a 2 -second image-acquisition delay. Posttreatment contrast-enhanced CBCT (Fig $1 D$ ) was performed as recently reported. ${ }^{16}$ Following stent-assisted coil embolization, the packing attenuation was determined by dividing the volume of coils implanted within the aneurysm (assuming each coil is represented by a solid cylinder) by the volume of the aneurysm as determined on 3DRA.

Although we routinely perform 3DRA during follow-up surveillance imaging, we preferred to use the immediate posttreatment CBCT to remove the effect of vessel remodeling that may occur with time from the analysis. To confirm that the geometric analysis from 3DRA and CBCT datasets were similar, we selected a single patient who had both studies performed immediately following stent-assisted aneurysm embolization. With the analysis method described below, the difference in the radii of curvature measured from the 3DRA and CBCT datasets was within $1 \%$. This test case was not included in the data series reported herein.

\section{Centerline Extraction}

Postimplantation CBCT images were exported in DICOM format and imported into Mimics 13.1 (Materialise, Ghent, Belgium) for segmentation and reconstruction. Segmentation was performed by using a gray value threshold to select only the struts of the stent followed by 3D reconstruction for visual inspection. Next, the Mimics built-in 3D LiveWire tool was used for isolation of the stent-containing vessel. The tool allows the separation and segmentation of small gray value changes that a broad threshold would not properly identify. From this final segmentation, a new 3D reconstruction was made of the stent-containing vessel segment and was subsequently smoothed to remove sharp corners introduced by the $3 \mathrm{D}$ algorithm (Fig $1 F$ ). Finally, the centerline of the vessel with control points every $0.4 \mathrm{~mm}$ was calculated, and these control points were exported to Matlab (MathWorks, Natick, Massachusetts) for re-sampling and curvature calculation (Fig $1 H$ ) as previously described. ${ }^{12}$

Pre-implantation 3DRA was similarly exported via the DICOM format and then imported into Mimics. However, with no stent present and no other artifact-inducing implants, the vessel segment was readily thresholded. The distances proximal and distal to the aneurysm were measured to isolate the vessel segment matching the postimplantation reconstruction. The path length was used to remove the measurement errors resulting from change in curvature. The segment was then smoothed, and the centerline was extracted as previously described (Fig 1E, $-G$ ).

\section{Curvature Calculation}

The same technique was used for both pre- and postimplantation curvature calculation as it was independent of the imaging technique. The centerline control points extracted from Mimics were imported into Matlab. The control points were resampled into an eighth degree polynomial at a spacing of $0.05 \mathrm{~mm}$. The resampling allowed any small error in centerline calculation or fluctuation to be removed from the curvature calculation. ${ }^{12}$ After resampling, both the original and resampled points were plotted on a graph to ensure that there were no errors during processing. Finally the curvature and the RC were calculated.

$$
\kappa=\frac{\left|r^{\prime}(s) \times r^{\prime \prime}(s)\right|}{\left|r^{\prime}(s)\right|^{3}}
$$

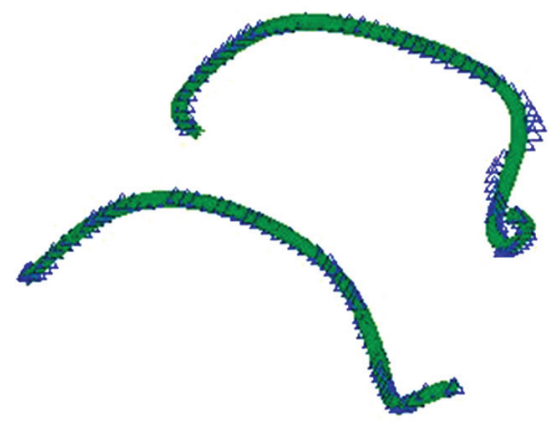

Fig 2. The best-fit polynomial (green line) of the resampled centerline data (blue triangles) is used to calculate the radii of curvature before (top) and after (bottom) stent implantation.

$$
R C=\frac{1}{\kappa},
$$

where $\kappa$ is the curvature, $r(s)$ denotes the location of a point along the polynomial representation of the centerline as a function of a single parameter $(r(s)=[x(s), y(s), z(s)]$ in rectilinear coordinates). $r^{\prime}(s)$ and $r^{\prime \prime}(s)$ are the first and second full derivatives, respectively. To better visualize the change in curvature, we graphed the pre- and post-implant centerlines on the same coordinate system (Fig 2). Finally, the average RC for each centerline was calculated. At the end of the vessel segments, 25 points $(1.25 \mathrm{~mm})$ were removed from either end of the centerline to eliminate the artifacts from the radiopaque markers of the stents.

\section{Phantom Experiments}

Phantom imaging experiments were performed to confirm that the analysis of data from 3DRA, and contrast-enhanced CBCT protocols allowed the reproducible calculation of vessel curvature. The phantoms consisted of 3 different patient-specific silicone vascular replicas of the ICA that were previously described ${ }^{12}$ suspended in $16 \mathrm{~cm}$ of water. The lumen of the replicas was flooded with pure contrast (iopamidol 51\%, Isovue), and 3DRA was performed per our standard clinical protocol. Immediately thereafter, CBCT was performed while injecting $20 \%$ by volume of contrast in saline into the replicas, per the clinical protocol. The data were processed as described above for the calculation of the curvature of the centerline. Specifically, the curvature of 4 segments of each ICA, namely the genu petrous, the retroGasserian petrous, the posterior cavernous genu, and the anterior cavernous genu, were recorded for both imaging datasets. A BlandAltman analysis was performed to establish the repeatability and reliability of the RC measurements with data from 3DRA and CBCT.

\section{Statistical Analysis}

Data were represented by the mean \pm SD and compared with a 2 -tailed paired $t$ test. A statistical significance level of $P<.05$ was used.

\section{Results}

A total of 12 arterial curves in 3 patient-specific vascular replicas were studied with both 3DRA and contrast-enhanced CBCT from the petrous segment to the distal aspect of the ICA siphon. The RC of the phantoms ranged from 3.9 to $11.8 \mathrm{~mm}$. The mean absolute difference between the RC calculations from these methods was $0.04 \pm 0.19 \mathrm{~mm}$ (Fig 3). A paired $t$ test of the RCs from 3DRA and CBCT revealed no statistical differences between these populations $(P>.05)$. On average, the CBCT method overestimated the RC by $0.34 \%$. 

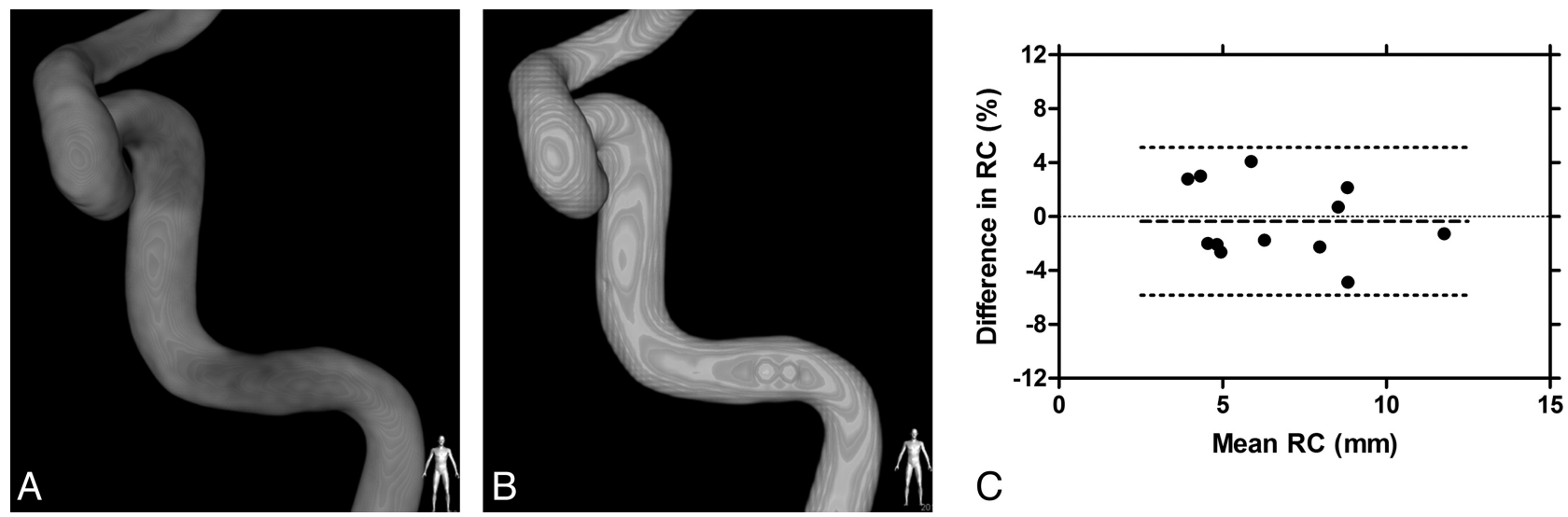

Fig 3. Rotational angiography $(A)$ and contrast-enhanced CBCT $(B)$ of 1 of the vascular phantoms. Bland-Altman plot $(C)$ of the differences in the radii of curvature measured based on data obtained from each imaging technique (bold dashed line is the mean difference; dotted lines are the limits of the agreement)

\begin{tabular}{lc}
\hline Patient demographics and aneurysm information & \\
\hline Demographics/Aneurysms & \\
\hline Patients & 24 \\
Aneurysms & 24 \\
Females & $18(75.0 \%)$ \\
Mean age (yr) & 49.5 \\
Age range (yr) & $18-68$ \\
Presentation/indications for treatment & \\
Headache/incidental & $15(62.5 \%)$ \\
Recurrent after coiling & $7(29.2 \%)$ \\
Stroke/transient ischemic attack & $2(8.3 \%)$ \\
Aneurysm dimensions & \\
Mean aneurysm dome size (SEM) (mm) & $5.6(0.8)$ \\
Mean aneurysm neck size (SEM) (mm) & $4.8(0.7)$ \\
Dome/neck ratio (SEM) (\%) & $1.2(0.09)$ \\
Aneurysm location & \\
ACA & $2(8.3 \%)$ \\
AcomA & $3(12.5 \%)$ \\
ICA & $16(67.7 \%)$ \\
ICA terminus & $3(12.5 \%)$ \\
\hline
\end{tabular}

Note:-SEM indicates standard error of the mean; AcomA, anterior communicating artery.

Of the 24 patients (Table), 21 received a closed-cell stent (Enterprise) and the remaining 3 received an open-cell stent (Neuroform). All patients received simultaneous coil embolization. The average unconstrained length of the implanted stents was $25.4 \pm 5.8 \mathrm{~mm}$. On completion of segmentation, reconstruction, and centerline extraction, the average pre-implantation centerline length was $28.5 \pm 6.0 \mathrm{~mm}$. The average postimplantation centerline length was $28.4 \pm 5.6 \mathrm{~mm}$. The 2 results were compared with a paired $t$ test, resulting in a mean difference of $0.02 \pm 2.3 \mathrm{~mm}$ and a $P$ value $>.5$, suggesting that there was no difference in the lengths of the segments.

The mean RC was $7.1 \pm 2.1 \mathrm{~mm}$ pre-stent implantation and $10.7 \pm 3.5 \mathrm{~mm}$ postimplantation (Fig $4 \mathrm{~A}$ ). The average RC was increased by $>50 \%$ from the values pre-implantation $(P<.0001)$. A histogram for the difference in curvature with a bin value set to $1 \mathrm{~mm}$ is shown in Fig $4 B$. Most stents were deployed in the ICA below its terminus. There was no difference in the change of the $\mathrm{RC}$ as a function of stent location (Fig $5)$. The change in $\mathrm{RC}$ was not dependent on the packing attenuation obtained within the aneurysm (Fig 6, $P>.05$ ).

\section{Discussion}

Aneurysm development and formation are, in part, due to a complex relationship between hemodynamics, vascular biology, and mechanical properties of the parent vessel. Sidewall aneurysms tend to form on the peak of curvature on the outside wall. ${ }^{10}$ It has also been shown computationally that with decreasing RC, the wall shear stress at the neck and within the aneurysm increases dramatically. ${ }^{17}$ The same study showed that a stent can reduce the wall shear stress of all but the most curved sections; however, it did not consider the effect that the stent would have on the curvature. Recently, research to determine which vessel characteristics might predict aneurysm rupture status ${ }^{11}$ has been reported by using a method similar to the one used here.

Previous studies have been performed to characterize the change in curvature of coronary vessels caused by stent implantation, ${ }^{13}$ and it is tempting to apply these results to intracranial arteries. However, the composition and environment of the arteries in the 2 different locations differ vastly as do the mechanical properties of coronary balloon-mounted stents versus self-expanding neurovascular stents. Compared with coronary arteries $(\mathrm{E}=16.8 \mathrm{kPa}, \nu=0.499),{ }^{18}$ arteries within the brain have different mechanical properties $(\mathrm{E}=9.2 \mathrm{kPa}$, $\nu=0.458) .{ }^{19}$ With a lower Young modulus and Poisson ratio, along with differences in the perivascular environment of the intracranial arteries, it can be assumed that larger deformation post-stent placement will occur compared with coronary arteries.

Based on our literature review, changes in the geometry of intracranial arteries as a result of stent deployment have been obtained from 2D angiograms of anterior communicating artery aneurysms. ${ }^{15}$ By measuring the angles between the $\mathrm{A} 1$ and the $\mathrm{A} 2$ on projection angiograms, Huang et al. found that the stent imposes a change in these angles, thus confirming stentinduced changes to the parent vessel. Although our results are not directly comparable because we describe the $3 \mathrm{D}$ change in the radius of curvature, the conclusion of significant alteration of the geometry of the native intracranial vasculature by stent implantation is confirmed by using the described 3D method. Other research has been conducted to determine the apposition of the stents within the tortuous intracranial arterial anatomy as a function of RC and parent vessel diameter, ${ }^{20}$ though 

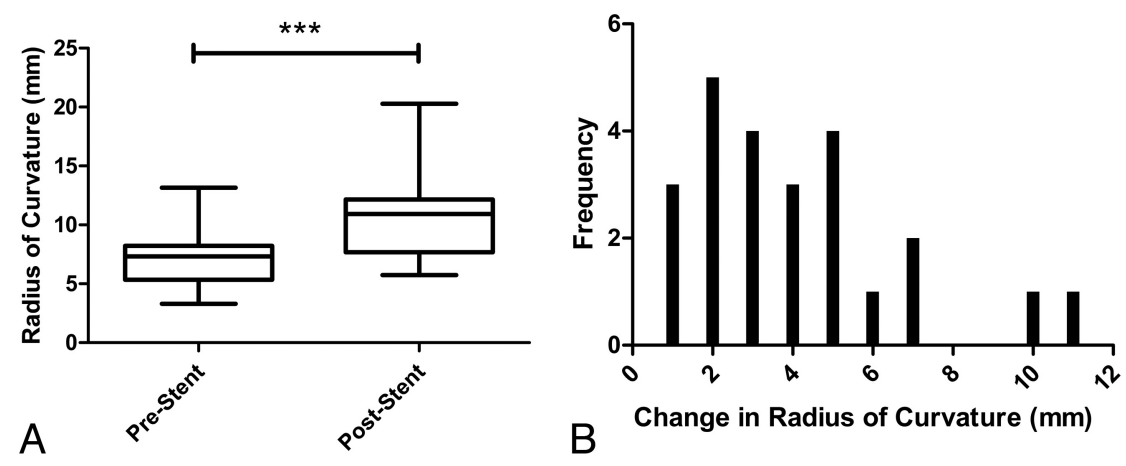

Fig 4. $A$, Box-and-whisker plots showing the radii of curvature before and after stent implantation $\left(^{* * *}, P<.0001\right.$, paired $t$ test). $B$, Histogram of the increase of the radii of curvature from pre- to post-stent implantation.

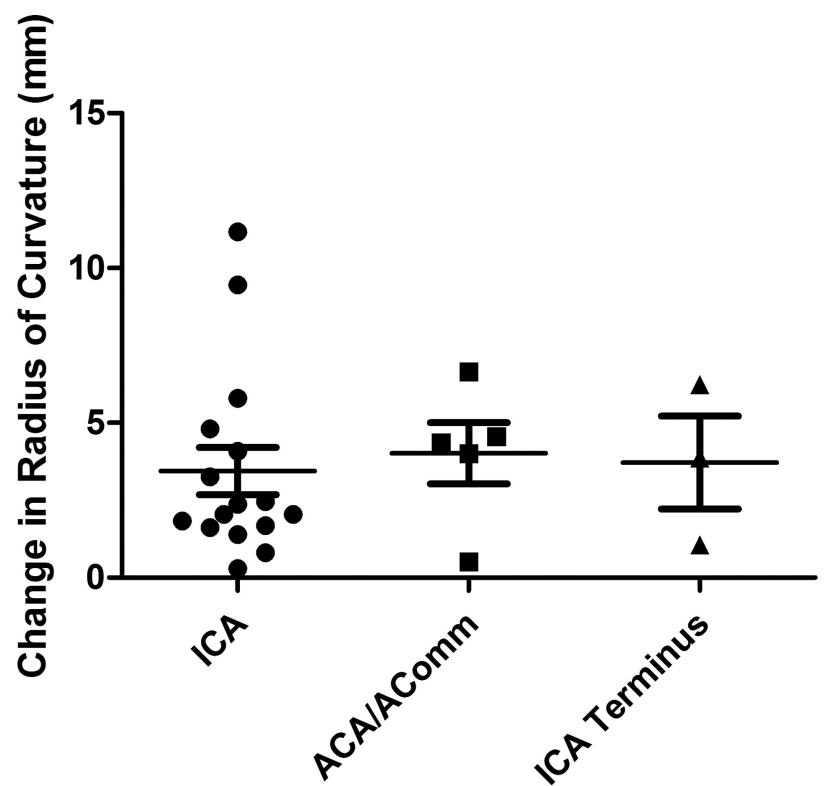

Fig 5. Box-and-whisker plot of the change in the radius of curvature due to stent implantation based on the anatomic location of the aneurysm.

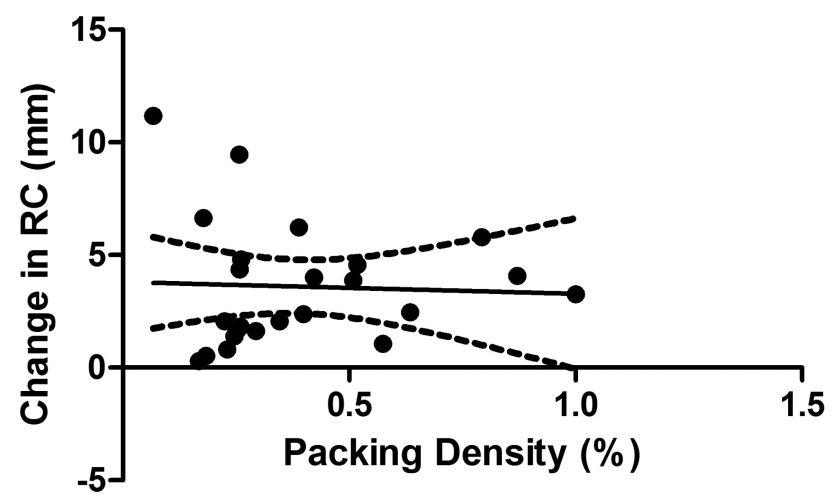

Fig 6. Linear regression analysis of the effect of packing attenuation on changes in the radius of the curvature of the stented segment $\left(P>0.05 ; R^{2}=0.002\right)$.

a comparison of the change in RC pre- and post-stent placement was not performed.

It has been shown in this study that the implantation of intracranial stents has a dramatic impact on the RC of the vessel. With the vessel straightening to a large degree, the local hemodynamics will presumably change as well. ${ }^{17}$ In the case of bifurcation aneurysms, the blood flow will change from an inertial-driven flow, which can cause much higher intra-aneurysmal kinetic energy and faster washout times, to a sheardriven flow as seen in sidewall aneurysms. ${ }^{21}$ This change in flow pattern should also change the locations of local higher wall shear stress, potentially aiding in the healing of the aneurysm. This result could be applied to the development of new devices designed to not only change the flow pattern but also remodel the vessel itself.

One of the study limitations was the use of 3DRA for preimplantation and contrast-enhanced CBCT for postimplantation imaging. Although we demonstrated that there was little difference between the calculations of vessel curvature from these 2 image-acquisition modalities, 3DRA has a slightly lower spatial resolution. Because there was no stent for isolation of the vascular segment pre-implantation, we chose to make measurements from anatomic landmarks such as the aneurysm. It is possible that small errors were introduced in identification of the vascular segment before stent implantation; however, because the path lengths of the pre- and postimplantation vascular segments were nearly identical, this is an unlikely source of significant error. In both the 3DRA and $\mathrm{CBCT}$, the aneurysm was artificially removed from the segmentation, potentially introducing error. Although we did not observe that the stent-induced changes in the radius of curvature were dependent on anatomic location, more data are needed to confirm this finding. Finally, our patient sample was heavily biased to closed-cell stent design, thereby inhibiting a comparison among different stent technologies.

\section{Conclusions}

In this study, it has been shown that not only do stents affect the curvature of intracranial vessels but that they do so to a degree far greater than in coronary arteries. These results can be combined with the results of CFD studies to help in the development of new flow-diverting stents and other intracranial devices. Often CFD studies assume that the vessel is a rigid nondeformable surface, and test the effect of stents on the original vessel. The reported results clearly demonstrate that this assumption is most likely too great a simplification.

Disclosures: Sarena Carniato-UNRELATED: Employment. Stryker Neurovascular. Ajay Wakhloo_-UNRELATED: Board Membership: Surpass Medical, Consultancy. Johnson \& Johnson, Codman Neurovascular, Stryker Neurovascular, Grants/Grants Pending: National Institutes of Health, Philips Healthcare, Payment for Lectures (including service on Speakers 
Bureaus): Harvard Medical School, Baptist Healthcare Miami, Stock/Stock Options: Surpass Medical, Travel/Accommodations/Meeting Expenses Unrelated to Activities Listed: Surpass Medical, Codman Neurovascular, Stryker Neurovascular, Covidien, ev3. Matthew Gounis—RELATED: Grant. Stryker Neurovascular, ${ }^{*}$ Comments: Research was supported by Stryker Neurovascular. I served as Pl; UNRELATED: Consultancy. Codman Neurovascular, Micrus Endovascular, Soteira, Comments: Consultant paid on a fee-per-hour basis; Grants/ Grants Pending: NIH, ${ }^{*}$ Concentric Medical, ${ }^{*}$ Stryker Neurovascular, ${ }^{*}$ Boston Scientific, ${ }^{*}$ eV3 Neurovascular, ${ }^{*}$ Codman Neurovascular, ${ }^{*}$ Sanofi-Aventis, ${ }^{*}$ Thrombolytic Science, ${ }^{*}$ Neuravi, ${ }^{*}$ Neurointerventional Therapeutics. ${ }^{*}$ ( Money paid to institution)

\section{References}

1. Wakhloo AK, Mandell J, Gounis MJ, et al. Stent-assisted reconstructive endovascular repair of cranial fusiform atherosclerotic and dissecting aneurysms: long-term clinical and angiographic follow-up. Stroke 2008;39:3288-96

2. Lylyk P, Cohen JE, Ceratto R, et al. Endovascular reconstruction of intracranial arteries by stent placement and combined techniques. $J$ Neurosurg 2002;97:1306-13

3. Jou LD, Mawad ME. Hemodynamic effect of Neuroform stent on intimal hyperplasia and thrombus formation in a carotid aneurysm. Med Eng Phys 2011;33:573-80

4. Maldonado IL, Machi P, Costalat V, et al. Neuroform stent-assisted coiling of unruptured intracranial aneurysms: short- and midterm results from a single-center experience with 68 patients. AJNR Am J Neuroradiol 2011;32:131-36

5. Mocco J, Snyder KV, Albuquerque FC, et al. Treatment of intracranial aneurysms with the Enterprise stent: a multicenter registry. J Neurosurg 2009;110:35-39

6. Bendok BR, Parkinson RJ, Hage ZA, et al. The effect of vascular reconstruction device-assisted coiling on packing density, effective neck coverage, and angiographic outcome: an in vitro study. Neurosurgery 2007;61:835-40

7. Lopes D, Sani, S. Histological postmortem study of an internal carotid artery aneurysm treated with the Neuroform stent. Neurosurgery 2005;56:416

8. Wakhloo AK, Schellhammer F, de Vries J, et al. Self-expanding and balloonexpandable stents in the treatment of carotid aneurysms: an experimental study in a canine model. AJNR Am J Neuroradiol 1994;15:493-502

9. Kulcsár Z, Urgon Á, Marosföi M, et al. Hemodynamics of cerebral aneurysm initiation: the role of wall shear stress and spatial wall shear stress gradient. ANJR Am J Neuroradiol 2011;32:587-94

10. Foutrakis GN, Yonas H, Sclabassi RJ. Saccular aneurysm formation in curved and bifurcating arteries. ANJR Am J Neuroradiol 1999;20:1309-17

11. Piccinelli M, Baciqaluppi S, Boccardi E, et al. Geometry of the internal carotid artery and recurrent patterns in location, orientation, and rupture status of lateral aneurysms: an image-based computational study. Neurosurgery 2011;68:1270-85

12. Chueh JY, Wakhloo AK, Gounis MJ. Neurovascular modeling: Small batch manufacturing of silicone vascular replicas. AJNR Am J Neuroradiol 2009;30:1159-64

13. Chen SY, Carroll JD, Messenger JC. Quantitative analysis of reconstructed 3-D coronary arterial tree and intracoronary devices. IEEE Trans Med Imaging 2002;21:724-40

14. Liao R, Green NE, Chen SY, et al. Three-dimensional analysis of in vivo coronary stent-coronary artery interactions. Int $J$ Cardiovasc Imaging 2004;20:305-13

15. Huang QH, Wu YF, Xu Y, et al. Vascular geometry change because of endovascular stent placement for anterior communicating artery aneurysms. AJNR Am J Neuroradiol 2011;32:1721-25

16. Patel NV, Gounis MJ, Wakhloo AK, et al. Contrast-enhanced angiographic cone-beam CT of cerebrovascular stents: experimental optimization and clinical application. AJNR Am J Neuroradiol 2011;32:137-44

17. Kim M, Taulbee DB, Tremmel M, et al. Comparison of two stents in modifying cerebral aneurysm hemodynamics. Ann Biomed Eng 2008;36:726-41

18. Baldewsing RA, deKorte CL, Schaar JA, et al. Finite element modeling and intravascular ultrasound elastography of vulnerable plaques: parameter variation. Ultrasonics 2004;42:723-29

19. Soza G, Grosso R, Nimsky C, et al. Determination of the elasticity parameter of brain tissue with combined simulation and registration. Int J Med Robot 2006;1:87-95

20. Heller RS, Malek AM. Parent vessel size and curvature strongly influence risk of incomplete stent apposition in Enterprise intracranial aneurysm stent coiling. AJNR Am J Neuroradiol 2011;32:1714-20

21. Augsburger L, Reymond P, Rufenacht DA, et al. Intracranial stents being modeled as a porous medium: flow simulation in stented cerebral aneurysms. Ann Biomed Eng 2011;39:850-63 